\title{
lohexol-measured glomerular filtration rate in children and adolescents with chronic kidney disease: a pilot study comparing venous and finger stick methods: response to comments from Dr. Luis-Lima
}

\author{
Amy Staples $^{1}$ (i) $\cdot$ Craig Wong $^{1} \cdot$ George J. Schwartz ${ }^{2}$
}

Received: 23 May 2019 / Revised: 23 May 2019 / Accepted: 31 May 2019 / Published online: 16 June 2019 (C) IPNA 2019

\section{Dear Editors,}

We read with interest the comments of Dr. Luis-Lima on our recently published article entitled "Iohexol-measured glomerular filtration rate in children and adolescents with chronic kidney disease: a pilot study comparing venous and finger stick methods" [1]. In our pilot study, we evaluated the agreement between glomerular filtration rate (GFR) measured with iohexol clearance via venous samples and samples collected via finger stick and dried blood spot (DSB) testing. We utilized a non-volumetric collection technique for our DBS collection and tried to apply only a single droplet of beaded up blood to each spot on the filter paper. Dr. Luis-Lima and his colleagues utilized both a non-volumetric and a fixed volume technique, collecting DBS samples by a capillary tube [2]. They demonstrated that the volumetric samples had better agreement than the non-volumetric method (total deviation index of $13 \%$ vs. $26 \%$ ), which improved to $9 \%$ (for the volumetric samples) with the addition of an internal standard, iopamidol.

We appreciate Dr. Luis-Lima's comment that the technique for collection of the samples (single collection for both venous and DBS testing) is unlikely to have affected their results. We agree that the volumetric collection of DBS would likely have improved our agreement. However, that was not the protocol we had developed for the pilot study; thus, our data is limited by the technique we used. In future studies utilizing the DBS technique for measuring iohexol-derived GFR, the technique described by Luis-Lima et al. should be considered superior to non-volumetric collection, even though it requires more handling by the phlebotomist.

We appreciate that Dr. Luis-Lima and his colleagues have noted this and allowed us to respond to their comments.

\section{References}

1. Staples A, Wong C, Schwartz GJ (2019) Iohexol-measured glomerular filtration rate in children and adolescents with chronic kidney disease: a pilot study comparing venous and finger stick methods. Pediatr Nephrol 34:459-464

2. Luis-Lima S, Gaspari F, Negrin-Mena N, Carrara F, Diaz-Martin L, Jimenez-Sosa A, Gonzalez-Rinne F, Torres A, Porrini E (2018) Iohexol plasma clearance simplified by dried blood spot testing. Nephrol Dial Transplant 33:1597-1603

Publisher's note Springer Nature remains neutral with regard to jurisdictional claims in published maps and institutional affiliations.
Amy Staples

astaples@salud.unm.edu

University of New Mexico, Albuquerque, NM 87131, USA

2 University of Rochester, Rochester, NY 14642, USA 